\title{
Application Study on Optical Fiber Network Technology in Communication Engineering
}

\author{
Li Li \\ College of Mobile Telecommunications Chongqing University of Posts and Telecom
}

\begin{abstract}
Keywords: Communication Engineering; Optical fiber network; Application advantage; Technical connotation
\end{abstract}

\begin{abstract}
With the development and progress of society, electronic information technology has made great progress where rapid development of optical fiber technology adds a thick and heavy color. Compared with traditional network technology, optical network technology has advantages of large capacity, fast speed, and strong anti-interference ability and so on. Therefore, in the future development of the network technology, optical fiber network will replace the traditional network, and the network technology will play a leading role which will also change people's way of life on the internet. This paper will mainly study the structure, advantages and application of optical fiber network technology of communication engineering.
\end{abstract}

\section{Introduction}

The arrival of a new era leads to the rapid development of information communication technology, people require the speed and quality of information communication which must be more and more high, but the speed of the traditional communication technology is slow with small capacity and weak anti-interference ability which cannot meet people's needs, so the optical fiber network technology homeopathy and health. O can effectively solve the weakness of traditional communication technology, such as it cannot overcome the difficulties, while optical fiber network technology can greatly improve the network communication technology in the past. In the future, in order to satisfy the needs of stability and efficiency of communication, the optical fiber network technology will be further developed to replace the traditional communication technology which is old, become the mainstream means of communication technology.

\section{Optical Fiber Network Technology}

Definition. In the optical fiber network technology, the fiber is a kind of medium which uses the fiber to carry on the light propagation, but the fiber mostly uses the glass or the resin to be made, and its principle is taken from the full radiation of the light. People transform the signal into optical signal propagation in the fiber on the one end, and then at the other end, through the optical signal receiving device converts light signals. In the process of propagation, the speed of light is very fast, and the interference is very small, so optical fiber network technology is very stable and efficient.

Composition of Structure. The structure of the optical fiber network includes a photoelectric conversion device, an optical signal receiving device, an optical fiber and a repeater. And the fiber is a communication cable composed of fibers. At both ends of the optical fiber, it respectively has the light signal transmitter and receiver, and the transmitter is used to transmit light signal, and the receiver is used to receive the light signal which can amplify the signal and detection signal. Photoelectric conversion equipment is the conversion of light signals into electrical signals or the electrical signals into light signals. These devices constitute the optical fiber network technology. Shown as the low figure: 


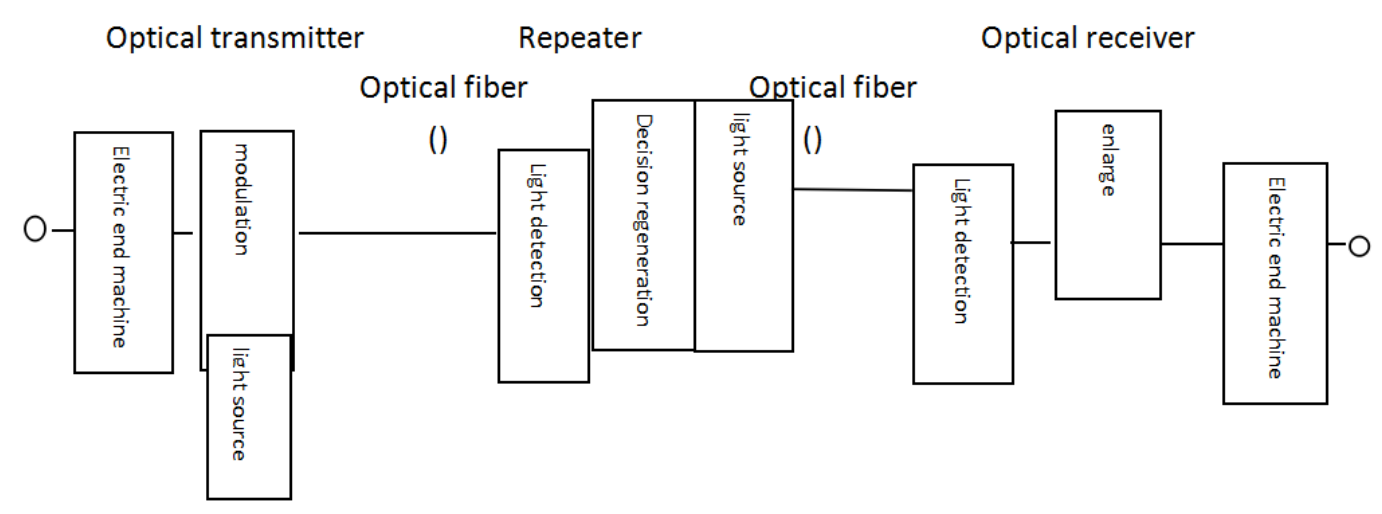

Figure 1

\section{Application Status of Optical Fiber Network Technology in Communication Engineering}

According to the statistical data, the development of China's optical fiber network has developed from the single mode optical fiber to the plastic optical fiber, and the cumulative use of optical fiber has been about $80000000 \mathrm{~km}$. The development of optical fiber technology leads to gradually increasing in fiber market in our country, then many businesses saw an opportunity in the market who have started to study excellent fiber which are faster with larger capacity and lower losses, and the development of fiber communication technology provides a good market prospect and technology support.

\section{Introduction of Advantage}

Strong Ability to Resist Electromagnetic Interference, High Stability. In the traditional communication, it always receive interference of electromagnetic wave, and the quality of communication signal seriously discounted, but with the development of wireless information technology, this factor cannot be effectively avoided, but be solved by adding some of the facilities to prevent electromagnetic interference, while these facilities will increase the cost, at the same time, it will also effect quality of transmission, and under such pressure, optical fiber technology develops. Optical fiber technology uses of transmission of light whose speed is very fast, and the light signal and the electromagnetic field is not directly related which will not affect electromagnetic field with the high stability, so the optical fiber network technology can reduce the transmission cost, and it also improves the communication quality.

Low Loss, Long Distance and Low Rate of Extraction. In the process of transmission, whether wireless or wired telecommunication number will increases the transmission distance, and the transmission distance is longer, this is a problem in signal propagation which is very difficult, which means it must add signal enhancement device based on the transmission way which will greatly enhance the investment and the cost of transmission. But in optical fiber technology, due to the propagation of light and the fast speed of light which can effectively avoid the occurrence of these problems. After testing, if use optical fiber network technology, it will reduce the signal attenuation per kilometer to the $0.25 \mathrm{db}$, and the attenuation value is very small, so it can greatly reduce establishment of equipment, and it also reduce the communication cost.

Small Leak, High Confidentiality. In practice, optical fiber communication engineering is much easier than the traditional communication engineering, because the signal of optical fiber communication weakened in the transmission process which is very small with less Signal amplification and enhancement device, at the same time, there is a glass fiber or resin composed of fiber with light weight and low requirements of the application environment. The sea mountains and rivers can be set, and the light in the optical fiber transmission cannot easily be read for signal, and it will also cut off communications interruption of fiber, this information will be sent out, and the signal cannot be stolen rather than the traditional signal as easy to be used in parallel mode to read signal. The special nature of the material and the characteristics of the optical fiber communication 
technology in the transmission process reduce the possibility of leakage of signals, and the confidentiality has also been increased.

Introduction of Disadvantage. Optical fiber has a series of advantages, and at the same time, there are also some disadvantages, such as low strength, brittle fiber properties, needing protection with high price, and the fiber cannot transmit power which needs special light source. Although there are so many disadvantages, but they don't affect the wide range of applications of optical fiber network technology in communication engineering, and with the development of technology and progress, these disadvantages can be effectively resolved.

\section{Introduction of Important Technology}

Network Base Station. In optical fiber communication engineering, network base station is the central engineering and information exchange hub which is an essential part. Due to the development of the communication network, the user of the communication network is rising exponentially, in order to adapt to the rising trend, the establishment of the network base station is essential. The communication base station and the decoding base station form a network base station. Network base station deals with the signal processing of the signal encryption, so that the information can be fully shared. Decoding the base station is used to interpret the light signal, and transform the optical signal into electrical signals, so that users can understand the transmission of information. The process is to encrypt the information to the base station, and the base station decodes the information into digital coding, and the results will be transmitted to the user to decipher. So the network base station plays an indispensable role in the transmission of the whole optical fiber technology which means it is the intermediate hub and core of the whole communication network.

Multiplexing Technology in Communication. In the process of communication of optical fiber network, not only $\mathrm{C}$ optical signal transmission, but also to deal with a variety of information and resources, it needs to use multiplexing technology. Multiplexing technology uses a variety of information collection in the same optical fiber processing which greatly improve the efficiency of the use of fiber, and spread as much information as possible in the same fiber. In order to maximize the use of optical fiber, it is to use the multiplexing technology to increase the multi-channel system to adjust the capacity of transmission media and so on. In the multiplexing technology, it is divided into many types, such as time, waveform, frequency, space and so on. Among them, waveform reuse can greatly improve the transmission quality of the communication engineering, and improve the utilization ratio of the optical fiber.

Dispersion Processing Technique. In the transmission process of fiber network communication, optical signal extinction of per kilometer is very few, but after investigation be found, after hundreds of thousands of kilometers, the loss of signal will be increased, and it may appear the distortion and garbled, so it needs to use dispersion processing technology. The dispersion can be enhanced processing technology of optical signal which can increase the expansion in the process of signal transmission model, enhance station distance, improve the ability to prevent signal interference and greatly reduce the attenuation of light signal in the transmission process to meet the signal transmission of time and distance requirements to ensure stability and speed long distance transmission.

\section{Application Foreground}

The prospect of application of optical fiber network in communication engineering

Fiber to the Home. Due to the development of society and the development of communication technology, there are more and more Internet users in China, but the fiber propagation speed is very fast which usually can reach hundreds of megabytes, so users demand for optical fiber is also growing. With the development and popularization of the technology of the optical fiber network, the cost of the optical fiber home is getting lower and lower. So far, China's economic development in the region has achieved relatively fast fiber to the home, but China's remote mountain areas and 
economically backward areas and the development of communication technology is slow, the communication efficiency is very low, but with the increase in the optical fiber network technology and people's needs, fiber network construction costs will be further reduced. Remote areas will also realize fiber to the home. Believe that "Fiber China" is coming on the road.

Develop all Optical Network. All optical network refers to the use of all the nodes in the optical fiber network to replace the traditional electric nodes to achieve full photochemical, in other words, the information has been used for light transmission and exchange which can greatly improve the efficiency of information transmission. At the same time, the photochemical also can improve the safety, reliability and compatibility of information transmission and it can greatly increase high network bandwidth, but also to prevent distortion in the process of information transfer and distortion free. From the current point of view, China's all optical network is still in the research stage, and no access to universal application, and it is not mature, but due to a series of advantages of all optical networks with the large space in use and development which has great potential for development, and it will become an important direction for future research and development.

\section{Conclusion}

In recent years, with the upgrading and progress of the optical fiber network technology, the fiber network goes into the public, and more and more people take it to the convenience. In order to reduce the cost of network construction with improving the efficiency of the use of network and improve the speed and quality of network communication, optical fiber network becomes essential. At the same time, because the fiber network's demand, the research on optical fiber network technology and technical personnel provide technical support for the development of optical fiber network which also let the fiber network in communication engineering in luminous splendor. In the future, the traditional network communication technology is gradually reduced, and the gradual popularization of optical fiber network will make the future of the world's network to be more advanced and scientific

\section{References}

[1] Dan Chen. Application of optical fiber network in communication engineering [J].Silicon Valley, 2014, 20:98+76.

[2] Lin Wang. Application of optical fiber network in communication engineering [J].Wireless Internet Technology, 2015, 03:7-8.

[3] Chengcheng Feng. Research on the application of optical fiber network in communication engineering [J].Electronic Technology and Software Engineering, 2016, 14:49.

[4] Xiang Li, Meiling Zhegn. Application of optical fiber network in communication engineering [J].Rural Economy and Technology, 2016, 18:264.

[5] Xiaoli Zhang. Research on the health structure monitoring system and reliability of sensor network of optical fiber [D].Nanjing University of Aeronautics and Astronautics, 2012. 